\title{
PMMA Cementoplasty in Symptomatic Metastatic Lesions of the S1 Vertebral Body
}

\author{
Amir R. Dehdashti, Jean-Baptiste Martin, Beatrix Jean, Daniel A. Rüfenacht
}

Neuroradiology-HUG, University Hospital of Geneva, CH-1211 Geneva 14, Switzerland

\begin{abstract}
We describe a lateral transiliac direct puncture approach to the S1 vertebral body for polymethylmethacrylate (PMMA) cementoplasty of painful metastatic lesions. This approach was performed using a $15-\mathrm{cm}$-long trocar needle with $3-\mathrm{mm}$ outer diameter, introduced under general anesthesia and fluoroscopic control. A lateral projection was used to center the needle just in front of the spinal canal and subjacent to the superior plate of the $S 1$ vertebral body. Needle progression was controlled using anteroposterior and lateral fluoroscopic projections alternately with a needle course parallel to an axial plane, avoiding conflict with the $S I$ foramen. After needle tip placement in the center of the SI vertebral body, diluted PMMA with a setting time of 8 min was delivered. Ipsilateral lesions of the lateral sacral compartment were filled with the same needle by stepwise withdrawal and continuous PMMA injection.
\end{abstract}

Key words: Percutaneous vertebroplasty-Sacrum metastasesPain treatment-Polymethylmethacrylate-Cementoplasty

The vertebral column is a frequent site for skeletal metastases [1], presenting often with pain due to vertebral body weakening. Vertebroplasty techniques, introduced a little over a decade ago (Deramond), are increasingly used to strengthen weak vertebral bodies by injecting polymethylmethacrylate (PMMA). Vertebroplasty allows for fast and durable pain relief for a variety of painful bone lesions with a success rate of $80 \%-90 \%$ and a complication rate of $2 \%-4 \%[2-4]$. PMMA injection is performed after transpedicular or direct lateral access to the vertebral body has been gained using a trocar needle. This approach, used for the lumbar and thoracic spine, exhibits difficulties at the SI level: the transpedicular approach does not allow the center of the vertebral body to be reached for geometric reasons and the direct lateral approach has the iliac wing in its way [5]. Direct access to the $S 1$ vertebral body would be possible coming from the lateral sacral compartment via a transiliac approach, as described below.

\section{Materials and Methods}

Three patients with painful metastatic lesions of the SI vertebral body were treated with PMMA cementoplasty after access was gained via a transiliac

Correspondence to: A.R. Dehdashti, M.D. approach. With the patient under general anesthesia and in the prone position a $15-\mathrm{cm}$-long trocar needle with $3-1 \mathrm{~nm}$ outer diameter (Trocart Vertebroplastie, Escoffier, Thonon-les-Bains, France) was introduced using fluoroscopic control. The procedure was started in the lateral fluoroscopic projection, centering the needle just in front of the spinal canal and subjacent to the superior plate of the SI vertebral body. Needle progression was controlled in the anteroposterior projection with a needle course parallel to an axial plane, above the level of the SI foramen, avoiding conflict with the nerve root (Fig. 1). The needle bevel was changed in its orientation such that the flat surface allowed for avoiding penetration toward structures such as the anterior surface of the sacral ala or the SI foramen. Along its trajectory, the beveled needle was rotated such that the sharp tip would initialiy help to penetrate the periosteum and the cortical bone layer and, once close to the S1 vertebral body, direct the needle tip toward the vertebral body center (Fig. 2). Additional CT control for needle guidance was used in one case [6. 71. PMMA (Simplex P, methylmethacrylate. Howmedica, Rutherford. NJ, USA) use involved the preparation of a diluted mixture $(20 \mathrm{ml}$ powder for $5 \mathrm{ml}$ liquid) allowing for an extended polymerization time of up to $8 \mathrm{~min}[4$, 8]. The PMMA powder was mixed with metallic powder to enhance its radio-opacity. Before injecting PMMA, vertebral phlebography allowed evaluation of the filling pattern and sites of potential PMMA leakage to be identified. Injection of opacified PMMA was performed under continuous visual control with fluoroscopy to obtain adequate filling and to avoid important PMMA leakage. Ipsilateral lesions of the lateral sacral compartment were filled with the same needle by stepwise withdrawal and continuous PMMA injection. To avoid unwanted deposition of PMMA in the gluteal soft tissues. the obturator of the trocar was introduced as soon as the needle tip reached the surface of the iliac bone and prior to complete withdrawal. Two cases received complementary filling by direct punctures of the lateral compartment using a posterior approach to the sacral ala. CT was used to identify adequate filling of the lesion before waking the patient. The filling was considered satisfactory in the presence of extension reaching from the superior plate of the vertebral body toward the sacroiliac joint (Fig. 3).

\section{Results}

The metastatic lesions of Sl vertebral bodies were treated in all three patients with good pain relief. Satisfactory filling of the S1 vertebral body was obtained using a transiliac approach in all three cases, and additional filling was required for lesion extension into the sacral alae in two cases. Contrast material injection prior to PMMA filling identified lesion necrosis in one case and viable tumor parenchyma aspects in all cases. Several points of potential leakage were identified and allowed for adaptation of PMMA injection or needle position. Learning about these leakage sites was also useful for directing attention to critical points observed during PMMA filling. A small venous leakage near the S1 radicular 

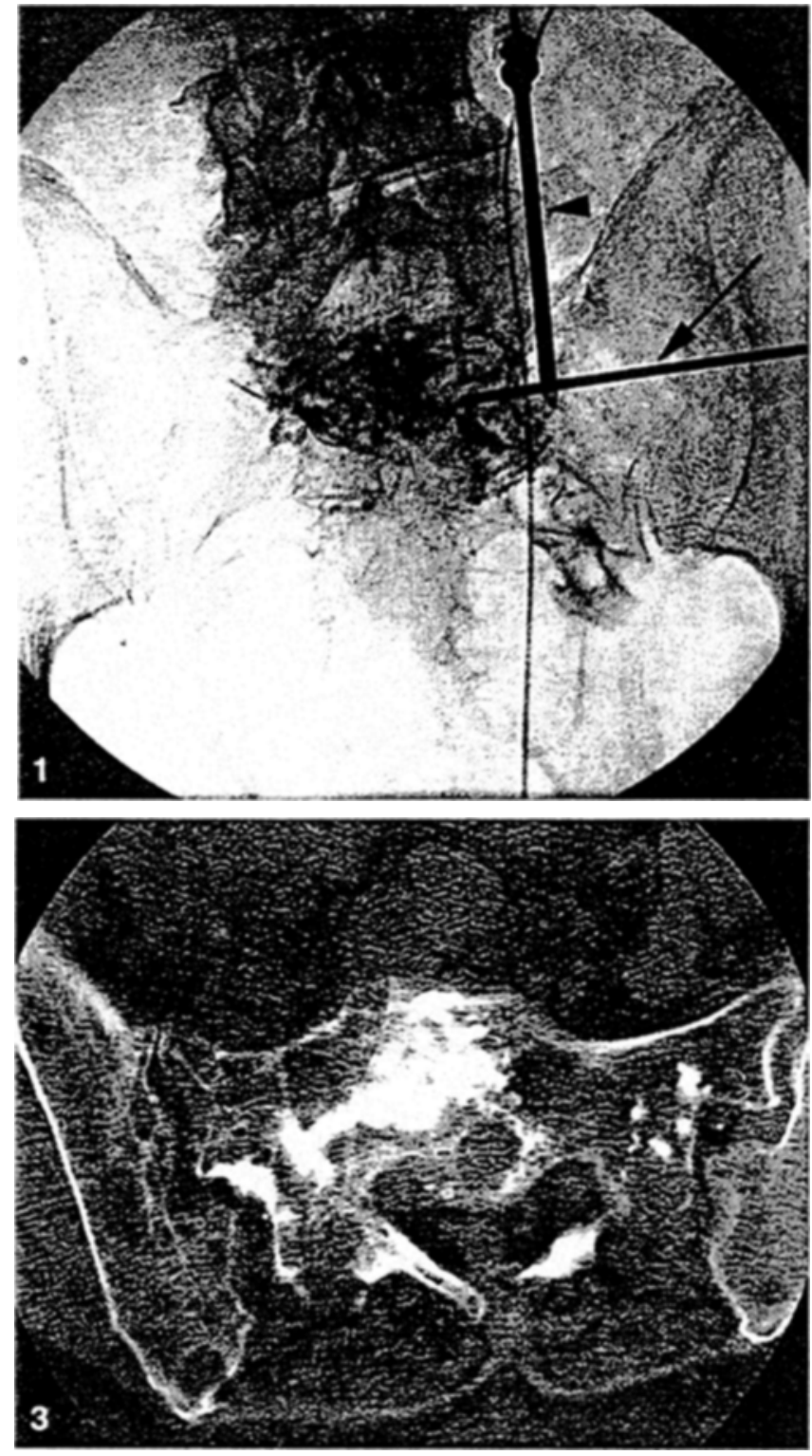

foramen was observed at the beginning of one PMMA injection. No other technical or clinical complication was observed.

\section{Discussion}

Vertebroplasty, an image-guided minimally invasive percutaneous treatment technique, enjoys increasing popularity due to its great potential to relieve pain rapidly and in a durable fashion in up to $90 \%$ of cases treated. In cases of vertebral metastasis, such pain palliation can be performed with a short hospital stay and with minimal patient discomfort [9]. On the basis of favorable experience in patients with this symptomatology. we extended the vertebroplasty technique to $S 1$ vertebral lesions and observed the same role of stability and antalgia. Having experienced difficulties in filling the $\mathrm{Sl}$ vertebral body by a transpedicular approach, we advocate the advantages of the transiliac approach; this allows for easy access to the vertebral body of $S I$ and filling of the sacral lesions from the midline toward the ipsilateral joint with one access.

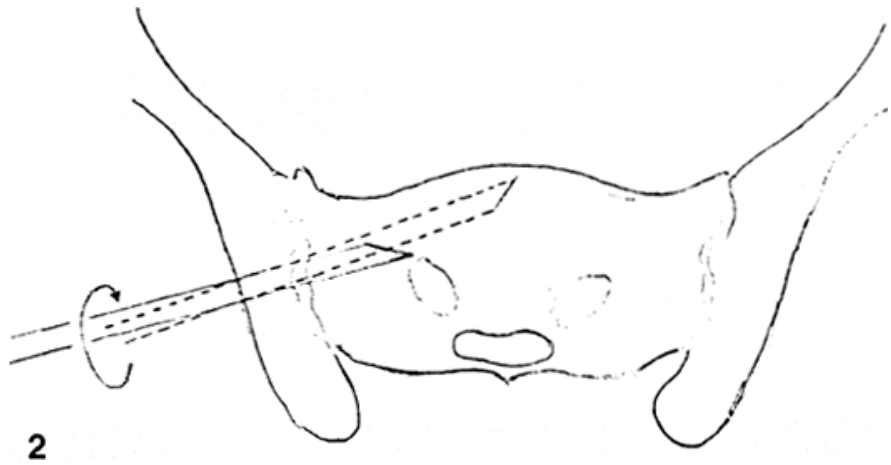

Fig. 1. Anteroposterior view of the pelvis showing a 2-mm outer diameter needle (arrow) introduced via a lateral transiliac approach. There is a needle in the ipsilateral sacral ala introduced via a posterior approach (arrowhead). Note the contrast material injected into the lesion revealing tumor parenchyma and draining veins (small arrowheads).

Fig. 2. Schematic drawing of the access depicting landmarks and needle rotations. Note the rotation of the sharp tip of the needle, once close to the $\mathrm{S} 1$ vertebral body, to avoid penetration toward the $\mathrm{S} 1$ foramen and to direct the needle tip toward the vertebral body center.

Fig. 3. Axial CT scan cementoplasty showing the PMMA implant with extension reaching from the vertebral body to the iliosacral joint.

The approach appears easy with reliable landmarks as seen on fluoroscopy and with a low risk for nerve root injury or aberrant access route.

Acknowledgment. The authors wish to express their thanks to Yasmine Sayegh, M.D. for preparing the schematic drawing of transiliac access.

\section{References}

1. Cotton A. Boutry N. Cortet B. Assaker R. Deramond H (1998) Vertebroplasty: State of the art. Radiographics 18:311-320

2. Cotton A. Deramond H. Cortet B (1996) Preoperative percutaneous injection of methyl methacrylate and $N$-butyl cyanoacrylate in vertebral hemangiomas. Am J Nucl Res 17:137-1+2

3. Weill A. Chiras J. Simon JM, Rose M. Sola Martinez T. Enkaoua E (1996) Spinal metastases: Indication for and results of percutaneous injection of acrylic surgical cement. Ridiology 199:2+1-2+7

4. Kalemmerlen P, Thiesse P, Bouvard H. Biron P. Mornex F. Jonals P (1989) Percutaneous vertebroplasty in the treatment of metastases: Technique and results. J Radiol 70:557-562 
5. Deramond H. Galibert P. Debussche C (1991) Vertebroplasty. Neuroradiology 33:392-394

6. Gangi A. Kastler BA. Dietemann JL (1994) Percutaneous vertebroplasty guided by a combination of CT and fluoroscopy. Am J Neuroradiol 15:83-86

7. Gangi A, Dietemann JL, Schuitz A, Mortazavi R, Jeung MY, Roy C (1996) Interventional radiologic procedures with CT-guidance in pain management. Radiographics 16:1289-1304
8. Martin JB, Jean B. Sugiu K, San Millan Ruiz D, Piotin M, Murphy K, Rüfenacht B, Muster M, Rüfenacht DA (1999) Vertebroplasty: Clinical experience and follow-up results. Bone 25 [Suppl 2]:11S$15 S$

9. Cotton A, Dewatre F, Cortet B (1996) Percutaneous vertebroplasty for osteolytic metastases and myeloma: Effect of the percentage of lesion filling and the leakage of methyl methacrylate at clinical follow-up. Radiology 200:525-530

\title{
Use of Through-and-Through Guidewire for Delivering Large Stent-Grafts into the Distal Aortic Arch
}

\author{
Muhammad Al Shammari, ${ }^{1}$ Peter Taylor, ${ }^{2}$ John F. Reidy ${ }^{1}$ \\ 'Department of Radiology, 2nd Floor, Guy's Tower, Guy's Hospital, St. Thomas Street, London SE1 9RT, UK \\ 'Department of Surgery, 2nd Floor New Guy's House, Guy's Hospital, St. Thomas Street, London SEI 9RT, UK
}

\begin{abstract}
The availability of large diameter stent-grafts is now allowing the endovascular treatment of thoracic aortic aneurysms. Most aneurysms are closely related to the distal arch and it is thus necessary to pass the delivery systems into the arch to effectively cover the proximal neck. Even with extra-stiff guidewires in position, it may still be difficult to achieve this, as a result of tortuosity at the iliac arteries and the aorta. We detail a technique where a stiff guidewire is passed from a brachial entry point through the aorta and out at the femoral arteriotomy site. This allows extra-support and may enable the delivery system to be passed further into the aortic arch than it could with just the regular guidewire position.
\end{abstract}

Key words: Aorta, aneurysm-Stents and prostheses

There has been only limited experience of stent-grafts in the thoracic aorta [1-4]. This has been principally due to the size limitations of available stent-grafts and also the fact that most aneurysms arise in close proximity to the origin of the left subclavian artery necessitating placement of the proximal portion of the stent-graft in the distal aortic arch. Advancing the large delivery catheter into the distal aortic arch can be difficult, even when a superstiff guidewire is well positioned in the ascending aorta. We have found that advancing the delivery device over a superstiff guidewire placed from the right brachial artery and out through the femoral artery makes it possible to achieve a much better position in the distal arch. We illustrate this technique with a case report.

\section{Case Report}

A 73-year-old woman who had sustained multiple fractures in a road traffic accident was noted on a chest radiograph to have a wide mediastinum, and subsequent chest CT scan confirmed extensive mediastinal hematoma. She was then referred to our hospital for further management. Aortography (Fig. 1) showed the typical appearance of aortic transection just distal to the left

Correspondence to: Dr. J.F. Reidy

DOl: $10.1007 / \mathrm{s} 002700010053$ subclavian artery origin with a pseudoaneurysm. In view of her age and associated problems, she was considered a high-risk patient for surgery and we were asked to consider her for stent-grafting.

The following day, in our endovascular radiology suite under general anesthesia, a right femoral arteriotomy was performed. Via a $4 \mathrm{Fr}$ sheath in the right brachial artery a catheter was passed through the transection into the abdominal aorta and right femoral artery. A $500-\mathrm{cm} \mathrm{0.035-inch} \mathrm{guide-}$ wire (superstiff guidewire, Schneider. Bulach, Switzerland) was passed through this and then grasped at the femoral arteriotomy. Sufficient guidewire was then withdrawn so that the stent-graft delivery system could be introduced over it through the femoral arteriotomy. When the delivery system was pushed into the distal arch, tension was maintained at both ends of the guidewire (Fig. 2). There was a concern that the guidewire could have a cutting effect on the proximal innominate artery, so a $4 \mathrm{Fr}$ catheter was advanced over it until its end was just in the aorta.

The stent-graft $(28 \times 60 \mathrm{~mm}$ AneuRx thoracic covered stent; AneuRx, Sunnyvale, CA, USA) was then deployed and its position was satisfactory, with the proximal end immediately distal to the left subclavian artery. It was necessary to deploy a second stent-graft $(28 \times 60 \mathrm{~mm})$ to cover the whole of the transection. A pigtail catheter was then advanced over the guidewire via the femoral approach. When the tip of the pigtail catheter was in the proximal arch the guidewire was withdrawn from the brachial approach through the brachial catheter, which was then removed. A post-deployment anrtogram (Fig. 3) showed a satisfactory position with exclusion of the pseudoaneurysm.

A spiral chest CT scan performed 5 days later revealed no evidence of leak with exclusion of the false aneurysm.

\section{Discussion}

Now that large-diameter stent-grafts are available, this technique is a very attractive alternative to surgery in the management of thoracic aortic aneurysms. As the majority of aneurysms are closely related to the distal arch, it is particularly important to place the proximal end of the stent-graft as close to the left subclavian artery as possible. Even with a stiff guidewire placed in the ascending aorta, there may be problems passing the large and relatively inflexible delivery system around the aortic arch. Using the through-and-through guidewire gives extra support and enables the delivery catheter to be pushed further and more safely into the aortic arch. 